\title{
Evaluating Existing Set-top Boxes Versus Transmit Diversity Schemes
}

\author{
Shirazi $\mathrm{H}^{1}$, Cosmas $\mathrm{J}^{1}$, Krishnapillai $\mathrm{K}^{1}$, Gledhill $\mathrm{J}^{2}$, Bard $\mathrm{M}^{3}$, Bradshaw $\mathrm{D}^{4}$ \\ 1. Brunel University, School of Engineering and Design, UB8 3PH, UK \\ 2. Videsco Ltd., UK, 3. Broadreach Systems, UK, 4. DTG Testing Ltd. UK \\ Hamidreza.Shirazi@Brunel.ac.uk
}

\begin{abstract}
Transmit diversity is a technique that exploits the nature of fading due to multipaths and that potentially incurs delay and pre- and post-echoes into the received signals. There is some concern that early set-top boxes would not be compliant in this respect. Also, introduction of such techniques that would degrade reception for inservice equipments would have an important impact, which should be evaluated. This paper presents a measurement campaign to establish whether the in-service DVB-T receivers are compatible with transmit diversity schemes and also to evaluate the possible diversity loss in the line-of-sight fixed receptions.
\end{abstract}

Index Terms-Delay Diversity (DD), DVB-T, Transmit Diversity, Threshold of Visibility (TOV), Set-top Box (STB), Line-of-Sight (LOS).

\section{PREFACE}

$\mathrm{T}$ he Multiple Input Multiple Output (MIMO) or diversity scheme is referred to a method for improving the quality and reliability of the message signal utilizing two or more communication channels with different characteristics. This scheme exploits the scattering effect of the channel by means of transmitting multiple signals in a controlled manner from different antennas and allowing independently faded signals to be detected at the receiver with or without the receive diversity. To date, transmit diversity is a commonly used technique for most digital mobile communications whereas it has not yet been applied to DVB systems.

The implementation of the receive diversity in the existing hand-held devices is sometimes impractical due to the problem of situating the antennas sufficiently far apart for UHF band reception. However, the transmit diversity (Multiple Input Single Output - MISO) does not impose these issues whilst still being compatible with the existing hand-held devices [1].

There are various classes of transmit diversity schemes such as Delay Diversity (DD), Cyclic Delay Diversity (CDD), Time Diversity, and Spatial Diversity wherein multiple versions of the same signal may be transmitted from different antennas and/or in different time instances. These techniques can be applied to DVB-T/H and DAB systems to increase the channel selectivity and reduce the deep flat fading probability [2], [3].

Simulation results reveal that such techniques would improve the quality of signal for Non-Line-of-Sight (NLOS) and worsen the Line-of-Sight (LOS) receptions [4]. Therefore, it is important for a broadcast operator to identify the reception difficulties due to the transmit diversity techniques and adjust the system to satisfy both LOS and NLOS receivers.

In this paper, the results of backwards compatibility tests conducted in the European PLUTO project [6] is provided to evaluate the diversity loss and performance of in-service settop boxes (STB) in the typical LOS situations. The performance of the receivers are analysed upon their capability to handle the multipaths conditions resembling the LOS fixed reception while applying the transmit diversity schemes.

\section{TRIALS BACKGROUND}

The test-bed architecture and configurations used in this laboratory trials were planned to closely simulate the real life conditions in a reproducible way. The MPEG-2 Transport Streams (TS) used in the evaluation were a simulated version of the signal received off-air. The Rohde \& Schwarz (R\&S) SFQ TV Tester was used to simulate Transmit Delay Diversity (DD) code (multipaths effects) and fading conditions in Constant Phase channel profile. The RF output of the TV Tester was led to a set of set-top boxes using a splitter. The STBs were a cross section of designs representing consumer and professional STBs widely used in the market. A TV-set connected to the system via a low-loss UHF/VHF switch was used to evaluate the performance of each STB. Figure 1 shows the block diagram and laboratory set-up.

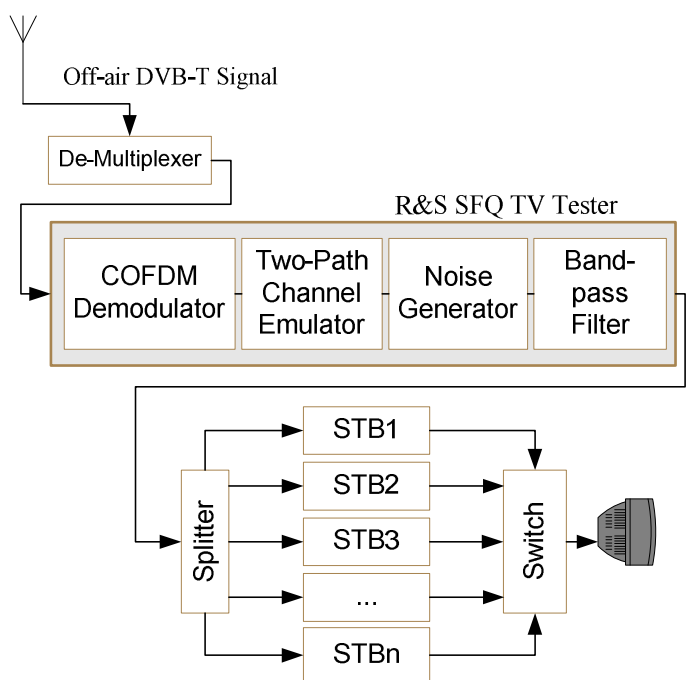

Figure 1: The laboratory set-up used in the test 
The off-air signal is of UHF Europe Channel 25 centred frequency 506MHZ which was re-modulated in R\&S SFQ TV Tester according to the DVB-T modulation schemes adopted by various European countries for Typical Urban (TU) environment [8]. Table I shows the modulation schemes used in the measurement campaign.

Table I: DVB-T modulation schemes

\begin{tabular}{cccccc}
\hline \hline Country & $\begin{array}{c}\text { OFDM } \\
\text { Mode }\end{array}$ & QAM & $\begin{array}{c}\text { Code } \\
\text { Rate }\end{array}$ & GI & $\begin{array}{c}\text { Guard } \\
\text { Delay } \\
(\mu \mathrm{s})\end{array}$ \\
\hline$U K$ & $2 k$ & 16 & $3 / 4$ & $1 / 32$ & 7 \\
$U K$ & $2 k$ & 64 & $2 / 3$ & $1 / 32$ & 7 \\
France & $8 k$ & 64 & $2 / 3$ & $1 / 8$ & 28 \\
Finland & $8 k$ & 64 & $2 / 3$ & $1 / 32$ & 112
\end{tabular}

In order to simulate the Transmit DD at the receiver headend, two paths was defined in the channel profile; tagged as "primary" and "echo". Each path was attributed in by different values of tap delays and mean powers. These conditions were simulated in SFQ TV Tester by setting values of path loss, delay and channel profile parameters. The echo signal was modelled as a low powered version of the primary signal arrived in different timestamp. Two scenarios were considered in the measurement campaign depending on the arrival times of the echo signal and primary signal at the receivers. The "Pre-echo" condition is defined when the echo signal arrives at the receiver prior to the primary signal, and "Post-echo" condition occurs when echo signal arrives after primary signal.

For each receiver, the minimum Carrier to Noise ratio $(\mathrm{C} / \mathrm{N})$ for decode-ability was determined by increasing the $\mathrm{C} / \mathrm{N}$ from $0 \mathrm{~dB}$, (with signal strength of $-46 \mathrm{dBm}$ including the cable loss of $-14 \mathrm{dBm})$ till the video was free of artefacts giving a "Threshold of Visibility" (TOV). The same process was then followed when Pre and Post-echo conditions were added.

Table II shows the relative delays and levels set in SFQ R\&S TV Tester for primary and echo signals during the measurement.

Table II: Primary and echo signals configuration.

\begin{tabular}{ccc}
\hline \hline $\begin{array}{c}\text { Test } \\
\text { cases }\end{array}$ & $\begin{array}{c}\text { Delay between } \\
\text { Primary \& Echo } \\
\text { Signals }(\mu \mathrm{s})\end{array}$ & $\begin{array}{c}\text { Relative Level of Echo } \\
\text { Signal to Primary Signal } \\
(\mathrm{dB})\end{array}$ \\
\hline 1 & No Echo & No Echo \\
2 & 0.5 & -10 \\
3 & 0.5 & -6 \\
4 & 0.5 & -3 \\
5 & 0.5 & 0 \\
6 & 1.0 & -10 \\
7 & 1.0 & -6 \\
8 & 1.0 & -3 \\
9 & 1.0 & 0
\end{tabular}

Table III shows the parameters set in R\&S SFQ TV Tester during the measurement campaign. The channel profile defined throughout the evaluation process to simulate fading situation, was "constant phase", which allows extremely short delays to be simulated. The other parameters like Doppler frequency and discrete component remained off during the experiments since they are used to simulate mobile reception and Rayleigh fading situations. The path loss and delay were values from test cases as presented in Table II. The Pre-echo and Post-echo conditions were studied according to the values set for paths 1 and 2 .

Table III: User-defined parameters used in the test

\begin{tabular}{lll}
\hline \hline $\begin{array}{l}\text { User-defined } \\
\text { Parameters }\end{array}$ & Path 1 & Path 2 \\
\hline Profile & Constant phase & Constant phase \\
$\begin{array}{l}\text { Path lost } \\
\text { Delay }\end{array}$ & \\
Speed $(\mathrm{m} / \mathrm{s})$ & Off & Off \\
Doppler frequency & Off & Off \\
Phase & O.0 DEG & O.0 DEG \\
Discrete & Off & Off \\
component & $0.0 \mathrm{~dB}$ & $0.0 \mathrm{~dB}$ \\
Power ratio & 1.0 & 1.0 \\
Frequency ratio &
\end{tabular}

\section{RESUlTS AND ANALYSIS}

In this section the results of the measurements are presented. Each test-case is separately analysed with regards to the level of visibility and reception perceived on a normal TV-set when applying a Transmit DD code to LOS set-top boxes (STB).

It is generally assumed that any signal arriving within the Guard Interval (GI) will not affect the receiver's ability to decode the signal. However, early evidence indicated that this might not always be the case, depending on the receiver's design and implementation. In reality, an echo signal arriving before the primary signal (pre-echo condition) would mean that it is arriving outside the GI, so a COFDM chip must actually lock to the pre-echo (weaker signal), and regards the primary signal as the interferer in order to function [10]. This would result in possible diversity loss and reception degradation.

\section{A. Test results in $U K-2 K$ mode, 16 QAM}

This section shows the performance of the STBs in Pre and Post-echo conditions where UK 16QAM 2k mode is applied. The TOV was investigated against the test cases explained in Table II. It was observed that the performance of the STBs deteriorated when transmit diversity scheme was applied via introducing the pre-echo condition.

Figures 2 and 3, respectively, show the reference behavior of STBs in Pre and Post-echo conditions for UK, 2K mode, 16 QAM. The test case numbered 1 shows the TOV level when no echo was introduced into the primary signal. In Fig. 2, the test cases numbered 2 to 5 shows the TOV level where primary signal was delayed by $0.5 \mu$ s from an echo signal leveled $-10,-6,-3$ and $0 \mathrm{~dB}$ relative to the primary signal. The test cases numbered 6 to 9 show the TOV level when the primary signal was delayed by $1 \mu \mathrm{s}$ from an echo signal leveled $-10,-6,-3$ and $0 \mathrm{~dB}$ relative to the primary signal. 


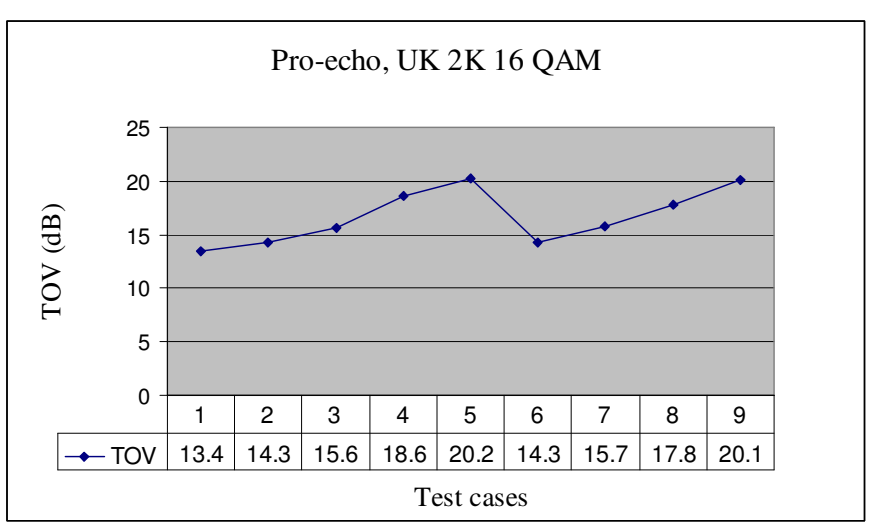

Figure 2: Pre-echo condition - UK 2K mode 16 QAM

Fig. 3 shows the TOV level where echo signal was delayed from the primary signal by 0.5 and $1 \mu \mathrm{s}$. The level of echo signal was set as described earlier (see also Table II).

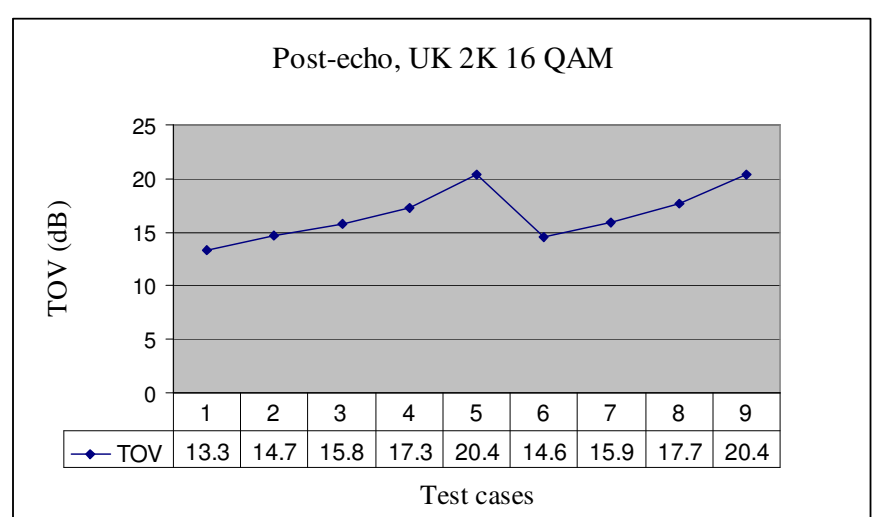

Figure 3: Post-echo condition - UK 2K mode 16 QAM

All receivers accepted the constellation variation; however an average diversity loss of $3.8 \mathrm{~dB}$ was experienced.

\section{B. Test results in $U K-2 K$ mode, $64 Q A M$}

In this section the performance of the STBs are shown where UK, $2 \mathrm{~K}$ mode with 64 QAM modulation scheme was used in SFQ TV Tester. Figures 4 and 5 show the TOV level in Pre and Post-echo conditions respectively; where an average loss of $3 \mathrm{~dB}$ was experienced.

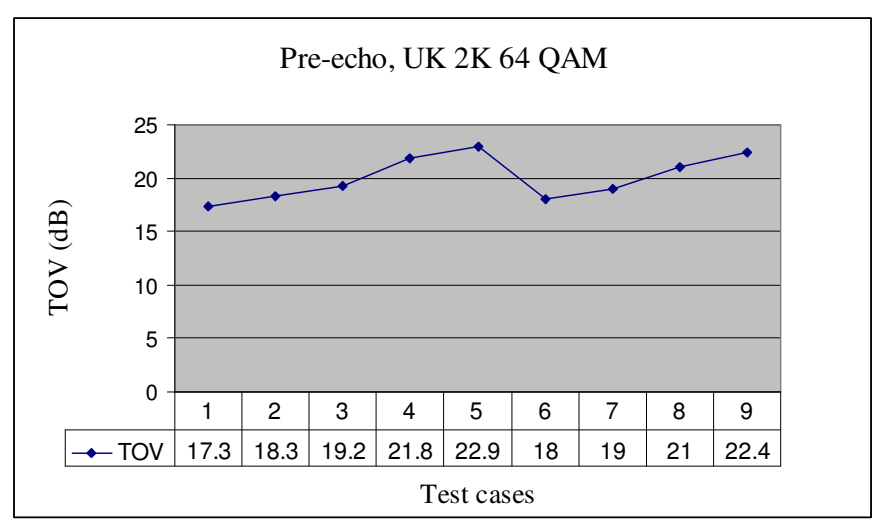

Figure 4: Pre-echo condition - UK 2K mode 64 QAM

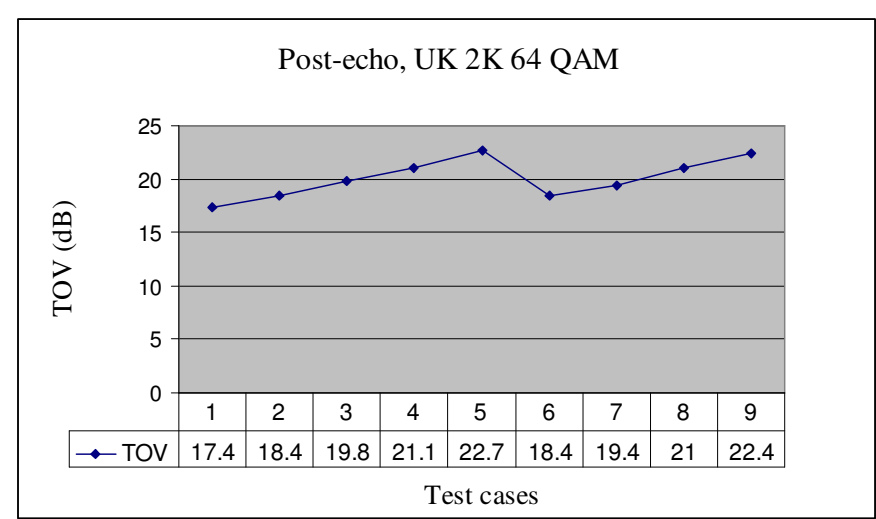

Figure 5: Post-echo condition - UK 2K mode 64 QAM

\section{Test results in France-8K Mode 64 QAM}

In this section the performance of the STBs are shown where France, $8 \mathrm{~K}$ mode with 64 QAM modulation scheme was applied. Figures 6 and 7 show the TOV level in Pre and Postecho conditions respectively; where an average loss of $2.3 \mathrm{~dB}$ was experienced.

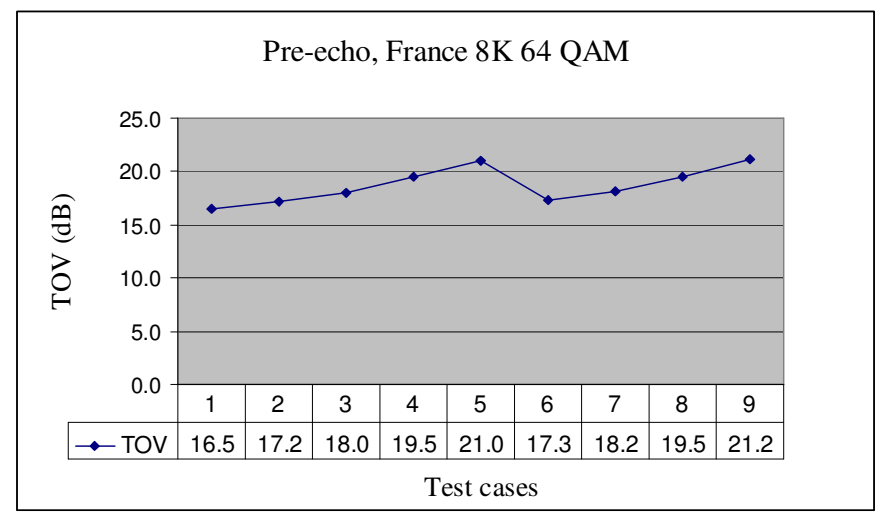

Figure 6: Pre-echo condition - France 8K mode 64 QAM

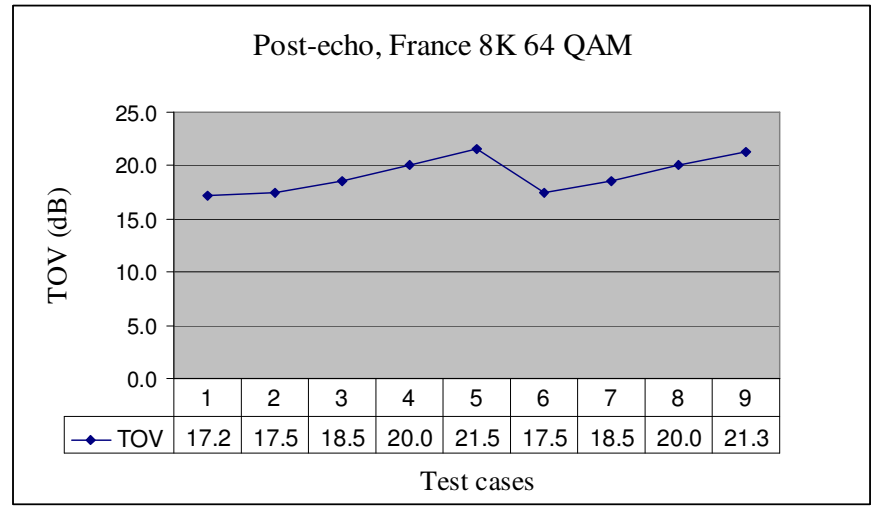

Figure 7: Post-echo condition - France 8K mode 64 QAM

\section{Test results in Finland-8K Mode 64 QAM}

In this section the performance of the STBs are shown where Finland, $8 \mathrm{~K}$ mode with 64 QAM modulation scheme was applied. Figures 8 and 9 show the TOV level in Pre and Post-echo conditions respectively; where an average loss of $2.2 \mathrm{~dB}$ was experienced. 


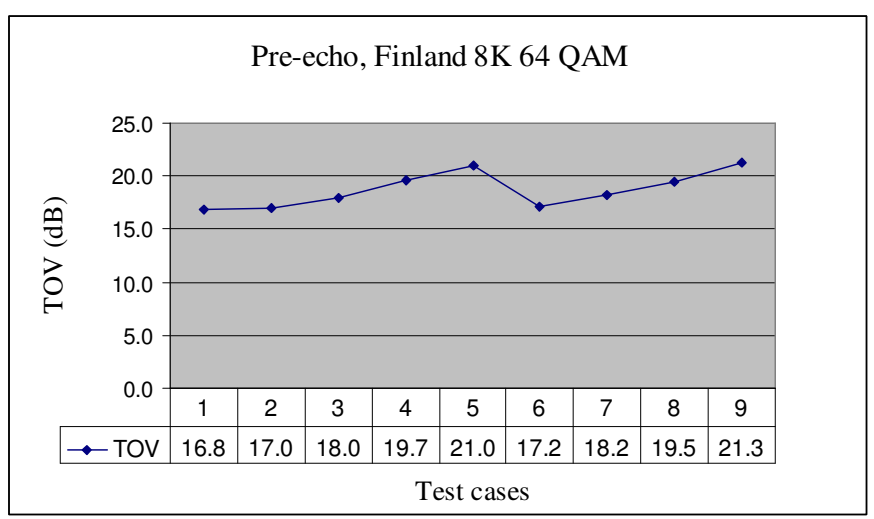

Figure 8: Pre-echo condition - Finland 8K mode 64 QAM

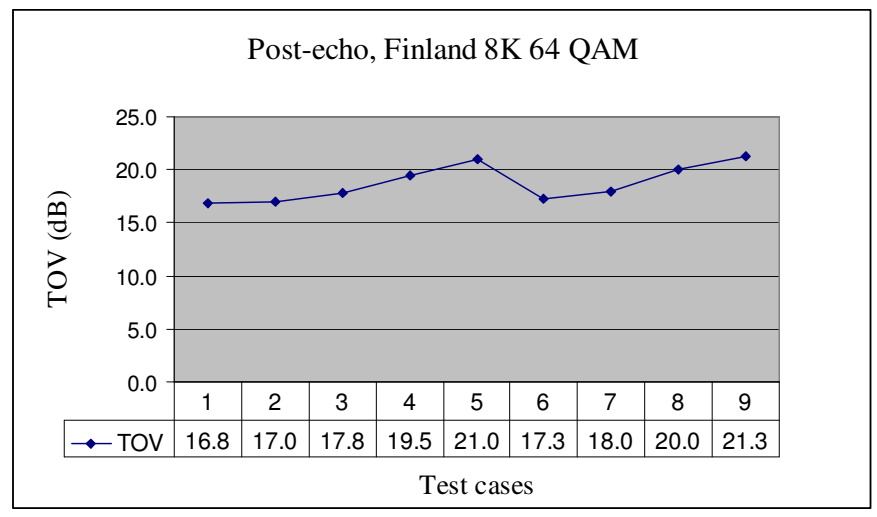

Figure 9: Post-echo condition - Finland 8K mode 64 QAM

\section{CONCLUSIONS}

This paper presents a measurement campaign to investigate whether early STBs are compatible with transmit diversity schemes (esp. DD, CDD). The diversity loss varies based on the modulation of signal, the strength of echo signal and the STB implementation. The increase $\mathrm{C} / \mathrm{N}(\mathrm{dB})$ required for successful decoding is due to the power of the echo signal $(10 \mathrm{~dB}, 6 \mathrm{~dB}, 3 \mathrm{~dB}$ and $0 \mathrm{~dB}$ below the primary signal) versus the relative timing of the echo. Whereas all the receivers accepted the constellation variation, an approximate diversity loss of $3 \mathrm{~dB}$ was experienced.

Results show that the multipaths effect of MISO would at worst cause TOV degradation of $7 \mathrm{~dB}$ in the $\mathrm{UK} 2 \mathrm{~K}$ mode and the 16 QAM modulation scheme on LOS fixed receptions when the level of echo signal equals to the level of primary signal. It is also deducted that STBs show more resiliency to multipaths defects when higher bit rate modulation schemes (i.e. Finland 8K mode, 64 QAM) are applied. In addition, higher degradation in the quality of signal and TOV is expected as echo signals become stronger; as a result, higher $\mathrm{C} / \mathrm{N}$ ratio is needed to preserve an intelligible picture.

During the laboratory trials, several receiver implementations were exhaustively tested under different test conditions to compare the response of different COFDM chipsets to MISO scheme in Ricean environments. The results of measurements can provide the STB manufacturer and broadcasters with guidelines to optimise STB implementations and transmit configurations to tackle the reception difficulties in case of using transmit diversity schemes for LOS fixed receivers.

\section{REFERENCES}

[1] H. Shirazi, R. D. Bari, J. Cosmas, J. Nilavalan, Y. Zhang, K.-K Loo, M. Bard, "Test-bed Development \& Measurement Plan for Evaluating Transmit Diversity in DVB Networks", Mobile and Wireless Communications Summit, $16^{\text {th }}$ IST, pp. 1-5, July 2007

[2] Y. Zhang, J. Cosmas, K.-K. Loo, M. Bard, and R. D. Bari, "Analysis of Cyclic Delay Diversity on DVB-H Systems over Spatially Correlated Channel" IEEE Trans. on Broadcasting VOL. 53, NO. 1, pp. 247-255, March 2007

[3] A. Dammann and S. Kaiser, "Standard conformable antenna diversity techniques for OFDM systems and its application to the DVB-T system," in IEEE Globecom, pp. 3100-3105,November 2001

[4] Armin Dammann, Ronald Raulefs. Report on Measurement Metrics and Theoretical Diversity Modelling. PLUTO Deliverable 4.1 August 2006

[5] S. M. Alamouti, "A Simple transmit diversity technique for wireless communications." IEEE J. Select. Areas Communications., vol. 16,pp.1451-1458, Oct. 1998

[6] "IST Project PLUTO," [Online]. Available: http://www.ist-pluto.org

[7] Commission of the European Communities. COST207. 1999. Digital Land Mobile Communications - Final Report.

[8] Digital Video Broadcasting (DVB); Framing structure, channel coding and modulation for digital terrestrial television (DVB-T). Draft European Norm EN 300 744, European Telecommunications Standards Institute ETSI.

[9] January 1999. Y. Zhang, J. Cosmas, M. Bard, and Y. H Song, "Obtain diversity gain for DVB-H by using transmitter/receiver cyclic delay diversity," IEEE Trans. Broadcasting, Dec. 2006, to be published.

[10] M. P. Brooks, A. Mattei, "DVB-T RECEPTION ISSUES IN A MOBILE ENVIRONMENT", Whitepaper [Online library:http://www.broadcastpapers.com/whitepapers/DV B-T-RECEPTION-ISSUES-IN-A-MOBILEENVIRONMENT$. \mathrm{cfm} ? \mathrm{objid}=32 \& \mathrm{pid}=601 \&$ fromCategory $=230$ ] 